\title{
Monte Carlo Modeling of Ion Beam Induced Secondary Electrons
}

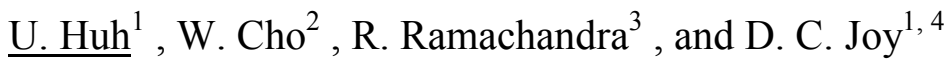 \\ 1. Material Science and Engineering, University of Tennessee, Knoxville, TN 37996-2200, USA \\ 2. Electrical and Computer Engineering, University of Tennessee, Knoxville, TN 37996-2100, USA \\ 3. National Center for Microscopy and Imaging Research, University of California San Diego, La Jolla, \\ CA 92093-0608, USA \\ 4. Center for Nanophase Materials Science, Oak Ridge National Laboratory, Oak Ridge, TN 37831, \\ USA
}

With the advent of high performance ion beam microscopes it becomes important to have access to methods which can predict, and interpret, the details of the ion beam interactions with the specimen and their contribution to the generation of the ion induced secondary electron (iSE) signal. Our initial work developed a Monte Carlo (MC) program, (IONiSE), for this purpose which performed well. However, in order for the MC code to generate quantitative data it is necessary to know the stopping power of the ion beam of choice in the target material of interest [1]. Data for the stopping power profile of $\mathrm{He}^{+}$ion beams in 39 elements, and a couple of binary compounds, was assembled from searches of the literature but, while this approach was successful, it was evident that it would be very time consuming, inconvenient, and challenging to assemble individual stopping power curves for all combinations of the incident ion and target material of interest.

We describe here an alternative approach. As a result of recent work by Berger et al [2] at NIST, the stopping power data required for the computation and interpretation of helium beam images can be generated using a computer program named "ASTAR". This program can compute the stopping power profiles for any ion impinging on an arbitrary target material, across five orders of magnitude of the beam energy. The ASTAR code is freely available at the NIST web site and can be run on any standard computer. The program plots the stopping power of the incident ion beam as a function of the energy and would normally be set up for a specific element. But, as shown in figure 1, if ASTAR stopping power data plots for incident helium ion beams are calculated for all the elements in the periodic table and then superimposed one on another it becomes clear that all the stopping power plots share not only the same general generic shape but, within the energy range from $10 \mathrm{keV}$ to $100 \mathrm{keV}$ which is of the most interest here, predict the stopping powers to also be very similar in their absolute magnitude. This effectively produces a "universal" stopping power curve for helium ion interactions with any given element and at any energies. With this stopping power data it is then possible for the IONiSE program to reliably predict parameters such as the beam range, and the secondary electron yields.

This approach can further be extended to the study of compounds irradiated by the incident helium ion beam. Figure 2 compares the ASTAR stopping power data plots calculated for a helium beam impinging on carbon and carbon dioxide, and also on silicon and silicon dioxide. Once again it can be seen that both the pure element, such as carbon, and a related corresponding binary compound, such as carbon dioxide, have stopping power profiles which are closely similar in their form and their magnitude. ASTAR stopping power data can therefore also be employed to model the stopping power and hence the iSE yield of complex multi-element materials. This approach provides a convenient solution to the problem of calculating iSE yields from compound materials, which so far has not been possible. 
Ideally, operators of helium ion microscopes would have immediate access to accurate and comprehensive ion stopping power, iSE yields, and other data, derived from careful experimental measurements. However, even after more than one hundred years of work with electron beams there is still no agreement on even such things as secondary electron yields as a function of beam energy and specimen composition, so it can be expected that gathering and analyzing comparable ion beam data will also be a long term problem. In such circumstances the simplified approach discussed here will continue to be useful.

\section{References}

[1] J. F. Ziegler. (2013). PARTICLE INTERACTIONS WITH MATTER. Available: http: //www .srim .org/

[2] M. J. Berger, J. S. Coursey, M. A. Zucker, and J. Chang. (2011). Stopping-Power and Range Tables for Electrons, Protons, and Helium Ions. Available: http: //www .nist.gov/pml/data/ star/index.cfm

[3] This works was partially supported by the Center for Materials Processing at the University of Tennessee.

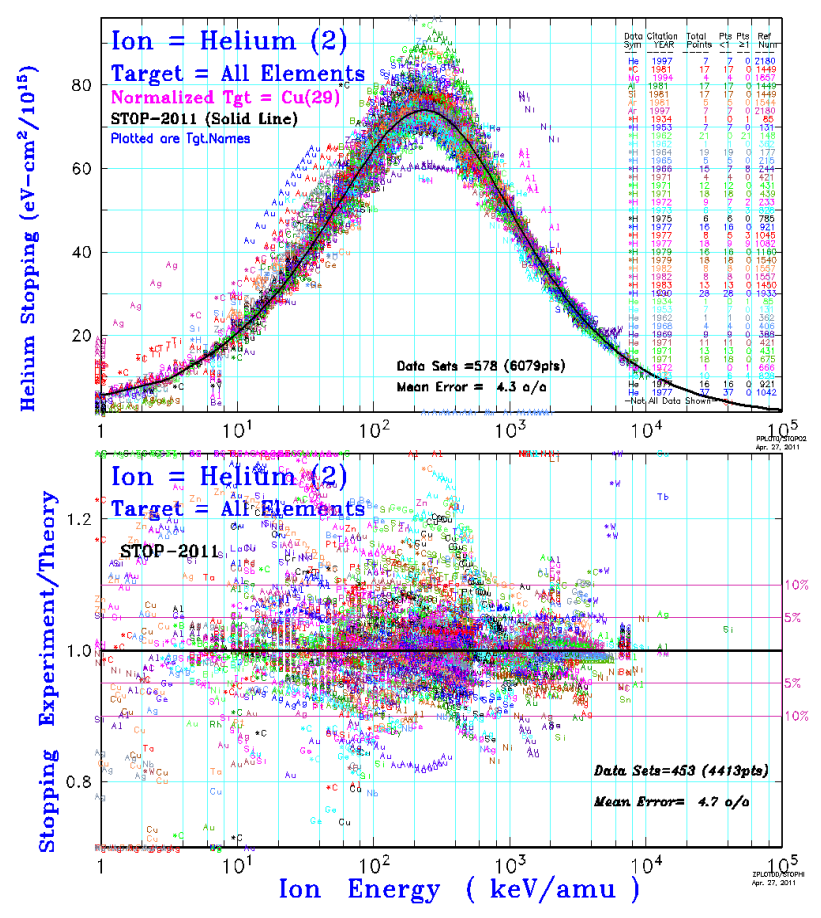

Figure 1. Universal ASTAR curve of helium stopping power as a function of ion energy [1]
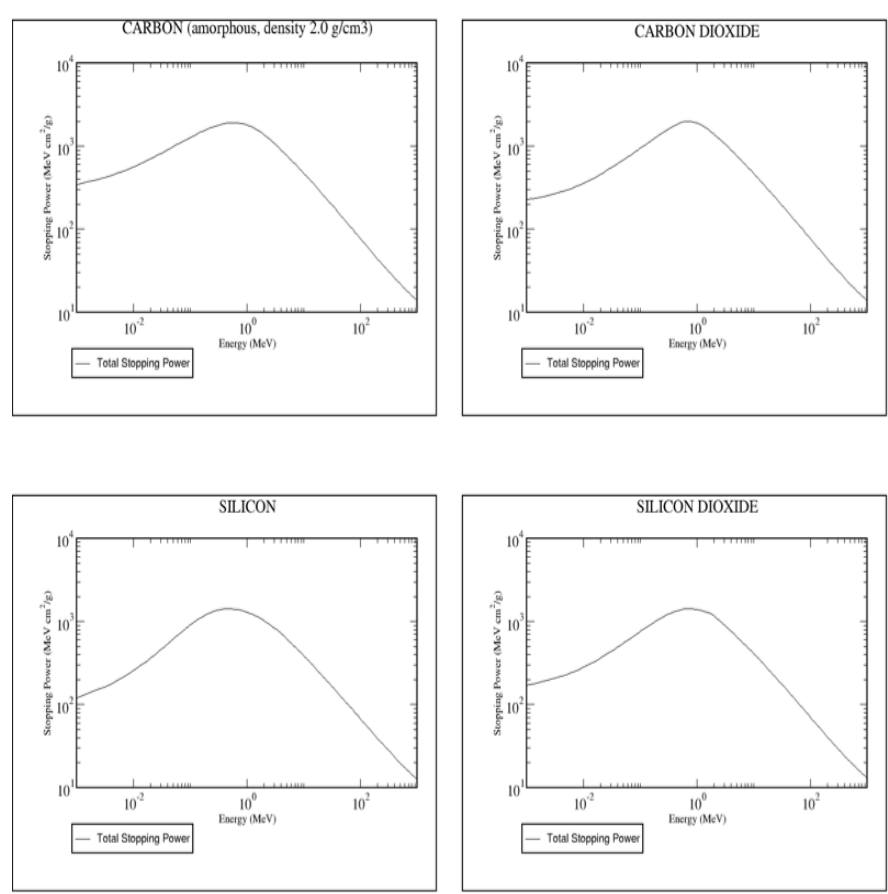

Figure 2. Stopping power of $\mathrm{C}, \mathrm{CO}_{2}$, $\mathrm{Si}$, and $\mathrm{SiO}_{2}$ for helium ions [2] 